\title{
Fisiopatología del colesteatoma originado a partir de un bolsillo de retracción
}

\author{
Physiopathology of the cholesteatoma originated \\ from a retraction pocket
}

\author{
Mauricio Cohen ${ }^{1}$, Claudio Callejas², Mauricio Salgado ${ }^{1}$
}

\begin{abstract}
RESUMEN
日 colesteatoma es una estructura quística delimitada por epitelio estratificado queratinizado con crecimiento expansivo. Se clasifican según su origen en congénitos y adquiridos. Los colesteatomas originados a partir de un bolsillo de retracción pertenecen a este último grupo. La patogénesis de su formación es multifactoria. Se inicia con la formación de un bolsillo de retracción en la que participan la disfunción de la trompa de Eustaquio y mayoritariamentelaneumatización de la mastoides. Numerosas teorías han tratado de explicar la transición desde este bolsillo de retracción hasta la aparición del colesteatoma Sudoff et al, al combinar la teoría de la invaginación y la de las células basales resuelve en parte este problema Sin embargo, deja en pie interrogantes como los mecanismos que desencadenan la migración celular y la proliferación celular. Esta es la barrera del conocimiento hoy en día Numerosas hipótesis han tratado de resolver esta interrogante, entre ellas, la hipótesis de las infecciones atípicas como los biofilms o la infección por $\mathrm{C}$ pneumoniae como agentes involucrados en la génesis de los colesteatomas originados a partir de bolsillos de retracción. En este camino de investigación han surgido novedosos conceptos como el uso del MIB1 yel MIF para uso potencial en poder predecir la agresividad y recurrencia de los colesteatomas.

Sin embargo, hasta ahora sólo se ha encontrado la presencia de infecciones atípicas en colesteatomas, pero no ha surgido ninguna línea de investigación que las correlacione con un aumento en la actividad celular, de manera de relacionarlas causalmente con una mayor agresividad clínica.

En este rumbo, recientemente la Sociedad Chilena de Aorrinolaringología ha otorgado financiamiento para un proyecto de investigación que buscará correlacionar la presencia de infección por $\mathrm{C}$ pneumoniae y una mayor agresividad clínica y molecular de los colesteatomas, medidas por MIB1. Esperamos que de esta manera se arroje mayor luz para comprender esta desafiante enfermedad.
\end{abstract}

\section{SUMMARY}

Cholesteatoma is a cystic structure delimited by stratified epithelium keratinized with expansive growth. They are classified according to their origin as congenital and

\footnotetext{
${ }^{1}$ Médicos del Servicio de Otorrinolaringología, Hospital San Juan de Dios, Universidad de Chile.

${ }^{2}$ Médico de la UDA de Otorrinolaringología, Pontificia Universidad Católica de Chile.
} 
acquired. Cholesteatomas originated from a retraction pocket belong to this last group. The pathogenesis of its formation is multifactorial. It begins with the formation of a retractable pocket in which disfunction of the Eustachian tube and majoritarily the pneumatization of the mastoid, participate. Numerous theories have tried to explain the transition from this retractable pocket to the appearance of the cholesteatoma. Sudoff et al, by combining the theory of invagination and that of the basal cells solves this problem in part. However, it maintains interrogations such as the mechanisms that start the cellular migration and cellular proliferation. This is the knowledge barrier to this date. Numerous hypothesis have tried to solve this question, among them, the hypothesis that atypical infections such as biofilms or infection due to Cpneumoniae as agents involved in the genesis of the cholesteatomas originated from retraction pockets. In this line of investigation several new concepts have risen as the use of MIB1 and MIF for potential use to predict the aggressiveness and recurrency of the cholesteatomas.

However, until now the presence of atypical infections has only been found in cholesteatomas, but no investigation line has appeared correlating them to an increase in cellular activity, in order to relate them casually to a higher clinical aggressiveness.

In this sense, the Chilean Society of Otolaryngology has recently granted financing for an investigation program that will seek to correlate the presence of infection by $C$ pneumoniae and a higher clinical and molecular aggressiveness of the cholesteatomas, measured by MIB1. This way we expect to obtain more information in order to understand this challenging disease.

\section{INTRODUCCIÓN}

Colesteatoma es un acúmulo anormal de epitelio escamoso queratinizado en un sitio anómalo, pudiendo, en el caso del oído, localizarse en oído medio, epitímpano, mastoides o ápex petroso. También ha sido definido como una estructura tridimensional, que exhibe crecimiento independiente, reemplazando mucosa de oído normal, y reabsorbiendo tejido óseo.

日 término "colesteatoma" fue acuñado por primera vez en 1838 por Johannes Müller para describir una neoplasia. Sin embargo, la estructura que hoy se conoce como colesteatoma no es una neoplasia, aunque comparte ciertas características como la capacidad de ser recurrente y potencialmente peligroso para el paciente.

Histológicamente se reconoce como un quiste benigno de células escamosas que consta de tres componentes: el contenido, la matriz y la perimatriz o lámina propia 8 contenido es el componente central y está formado por escamas de queratina completamente diferenciadas, acelulares. La matriz es el componente intermedio y está formada por epitelio escamoso queratinizado y da sostén a la estructura quística. La perimatriz está formada por tejido de granulación y puede o no tener cristales de colesterol. Está en contacto con tejido óseo y es esta perimatriz la que produce las enzimas proteolíticas que pueden provocar destrucción ósea ${ }^{1,2}$.

Aunque puede cursar en forma asintomática, muchos colesteatomas se manifiestan clínicamente como hipoacusia y otorrea $\mathrm{Si}$ la enfermedad progresa puede llevar a la parálisis facial, disfunción vestibular, e incluso complicaciones intracraneales como abscesos cerebrales y meningitis entre otras, siendo todas estas manifestaciones debidas en gran parte a su capacidad de destruir hueso.

Una vez establecido el colesteatoma su único tratamiento es la erradicación quirúrgica y posterior intento de reconstrucción del oído. 


\section{CLASIFICACIÓN}

Los colesteatomas se han clasificado según su etiología en congénitos o en adquiridos, los cuales, a su vez, se pueden dividir en primarios o secundarios.

En relación a los congénitos, se piensa que surgen de inclusiones embrionarias o restos de células epiteliales. Se encuentran detrás de una membranatimpánica intacta, no tienen continuidad con el conducto auditivo externo y no se reconocen factores etiológicos como perforaciones de membrana timpánica o historia previa de infecciones de oído. Estos a su vez se clasifican según su localización en el hueso temporal pudiendo localizarse en la pirámide petrosa, mastoides u oído medio.

Levenson y Michaels ${ }^{3}$ establecieron en 1989 una serie de criterios para el diagnóstico clínico de colesteatoma congénito: (1) masa blanca medial a la membrana timpánica, (2) pars fláccida y tensa de aspecto normal, (3) sin historia de otorrea o perforaciones, (4) ausencia de antecedentes de procedimientos quirúrgicos otológicos y (5) el antecedente de otitis media aguda no es criterio de exclusión. En su estudio donde incluyeron a 40 pacientes la edad promedio de presentación fue de 4,5 años, con una preponderancia por el hombre de 3:1, situándose el colesteatoma en el cuadrante anterosuperior en dos tercios de los casos.

日 colesteatoma adquirido primario es el que se origina a partir de un bolsillo de retracción y son los más frecuentes observados en la práctica clínica. Generalmente involucran la región del ático y se originan a partir de la pars fláccida. Sadé y Halevy 4 en 1985 describieron cuatro etapas de la retracción timpánica en relación a la pars tensa: (1) membrana retraída, (2) retracción contacta la apófisis larga del yunque, (3) atelectasia timpánica y (4) otitis media adhesiva.

En relación a la pars fláccida, Tos y Poulsen ${ }^{21}$ clasificaron las retracciones en: grado I, retracción hacia el cuello del martillo, pero sin tocarlo; grado II, retracción que contacta el cuello del martillo; grado III, retracción que sobrepasa el annulus óseo, con fondo visible y grado IV, con reabsorción del annulus óseo con retracción que llega a la cabeza del martillo.
En bolsillos de retracción profundos la queratina no migra normalmente, acumulándose. Son estos bolsillos de retracción los considerados como precursores de colesteatoma.

\section{ANATOMÍA Y EXPANSIÓN}

Los colesteatomas se expanden con patrones de crecimiento bastante constantes. Son canalizados a través de vías características por los pliegues de mucosa circundantes, la cadena osicular y sus ligamentos suspensorios. Un conocimiento de la anatomía del oído en general y de la pars flácciday ático en particular es fundamental para entender su progresión y las bases de su manejo quirúrgico.

Los colesteatomas epitimpánicos se originan a partir del espacio de Prussack situado entre la pars fláccida y el cuello del martillo. Sus sitios de expansión más frecuentes en orden descendente son a través del epitímpano posterior, mesotímpano posterior y el epitímpano anterior. A través del epitímpano posterior el coleteatoma logra acceso a la mastoides a través del aditus ad antrum. Por el mesotímpano posterior logra acceso a la región de la platina del estribo, el seno timpánico y el receso facial. La vía del epitímpano anterior lleva hacia el nervio facial y el receso supratubario, y puede pasar desapercibida durante la timpanoplastía si no se localiza dirigidamente.

\section{FISIOPATOLOGÍA}

La fisiopatología de los colesteatomas originados a partir de un bolsillo de retracción es controversial. A lo largo de años de investigación se han establecido numerosas hipótesis algunas más aceptadas que otras. Describiremos las teorías más aceptadas de la formación de este tipo de colesteatoma, desde las más básicas a las más novedosas y de vanguardia.

\section{A. Conocimiento básico}

La teoría de la invaginación (Vittmaack 1933) es considerada como uno de los mecanismos inicia- 
les en la formación de los colesteatomas de bolsillos de retracción. Esta sostiene que el proceso se inicia con la disfunción de la trompa de Eustaquio. La fluctuación repetida entre presión positiva y negativa en el oído medio asociada a procesos inflamatorios que se producirían intercurrentemente en el espacio de Prussack, llevarían a la pérdida del soporte estructural de la membrana timpánica y a su subsecuente retracción. La pars fláccida al ser la parte más distensible de la membrana es la más susceptible de retraerse. De no resolverse, la alteración en la migración de la queratina que se produce en el interior del bolsillo, llevará a su acumulación con la subsecuente formación de colesteatoma

Chole y Tinling 5 en 1985 ligando la trompa de Eustaquio a ratones de experimentación logran reproducir colesteatomas, lo que se transforma en la primera evidencia científica de que los colesteatomas pueden surgir de retracciones en la membrana timpánica.

Poe y Abou-Halawa ${ }^{6}$ en 2001 publican un análisis objetivo de la función de la trompa de Eustaquio utilizando video de baja velocidad en 64 pacientes. Se evaluó función muscular, grado de enfermedad de la mucosa, obstrucción de la mucosa y grado y frecuencia de apertura de la trompa. Observaron que todos los oídos con patología ya sea otitis media con efusión o colesteatoma presentaban grandes alteraciones de la función tubaria. Sin embargo no hubo correlación entre severidad de la enfermedad y grado de disfunción tubaria Este trabajo además demostró que el estudio de la función tubaria con video de baja velocidad, es una técnica potencialmente útil para clasificar los tipos de cambios patológicos en la trompa de Eustaquio.

日 grado de neumatización de la mastoides también juega un rol en la presión negativa del oído medio y la subsecuente formación de bolsillo de retracción. Es así como se ha observado que la mastoides actuaría como un buffer de presión dentro del oído medio. En 1997, Sadé y Fuchs ${ }^{7}$ demostraron una correlación inversa entre grado de neumatización de la mastoides y retracción de la pars fláccida Sadé en 2001 describe el fenómeno que denomina como hiperectasis en que la presión en el oído medio se eleva sobre la atmosférica en oídos con mastoides poco neumatizadas, lo que lleva a la protrusión de la membrana hacia el conducto auditivo externo. Hasebe et $\mathrm{al}^{9}$ en 2001 observaron a tres grupos de pacientes: uno con bolsillo de retracción (pacientes con retracción atical por más de 12 meses), otro grupo no quirúrgico (pacientes con colesteatoma que pudieron ser manejados sólo con tratamiento conservador por más de 12 meses), y un grupo quirúrgico (pacientes que requirieron cirugía en menos de un año a pesar de haber recibido tratamiento conservador). Observaron que la aireación adecuada de la mastoides estaba presente en 85 y $75 \%$ del primero y segundo grupo respectivamente y en $26,5 \%$ del tercero. No hubo diferencias en la función de la trompa de Eustaquio. Lo anterior sugiere que la progresión del colesteatoma se debe mayoritariamente a las condiciones de la mastoides y no de la trompa de Eustaquio. Además sugiere que el tratamiento conservador con tubos de ventilación sólo sería posible frente a mastoides aireadas.

Las teorías anteriores nos explican la formación del bolsillo de retracción, pero aún falta comprender cómo a partir de él se forma un colesteatoma.

\section{B. Conocimiento avanzado}

La teoría de la metaplasia sostiene que la infección recurrente o crónica del oído medio puede llevar a la transformación de su epitelio cuboideo simple a epitelio estratificado queratinizado. Esta teoría se fundamenta en el conocimiento de fenómenos de metaplasia a raíz de inflamación crónica en otros sitios corporales como el epitelio del tracto digestivo y del aparato respiratorio. Sadé tomó biopsias de oído medio en niños con otitis media encontrando islotes de epitelio queratinizado. Chole y Frush (1982) encontraron que deficiencias de vitamina A pueden llevar a la formación de epitelio queratinizado en el oído medio. Sin embargo, puntos en contra de esta teoría son el hecho que ningún estudio ha encontrado acumulación de escamas córneas y que clínicamente existe poco apoyo para el papel de la metaplasia en la formación de colesteatomas de bolsillos de retracción.

Una tercera teoría contempla el avance de piel a 
través de una perforación de la membrana timpánica, es así como esta perforación ocurre principalmente en el contexto de una otitis pero también puede ocurrir como consecuencia de un trauma. La destrucción del epitelio cuboideo a causa de la inflamación puede llevar a la migración de células del epitelio estratificado sobre la superficie denudada del oído medio, en un proceso que se conoce como estimulación de contacto. Al contactarse este epitelio con el epitelio normal del oído medio se produciría la detención de la migración en un proceso que se conoce como inhibición de contacto. Por alguna razón esta detención natural no se produciría, llevando a la formación de un colesteatoma Sin embargo, al igual que la teoría anterior, esta teoría parece tener un rol limitado en la formación de los colesteatomas a partir de bolsillos de retracción.

Una cuarta teoría propone el crecimiento del epitelio queratinizado hacia la lámina propia de la membrana timpánica lo que correspondería a una especie de invasión epitelial. La lámina o membrana basal es una barrera entre el epitelio y la lámina propia (tejido conjuntivo). Esta teoría llamada de inclusión papilar o de las células basales sostiene que roturas de la membrana basal permitirían el crecimiento del epitelio estratificado hacia la lámina propia. Estas roturas estarían originadas por procesos inflamatorios que ocurrirían en el espacio de Prussak. Chole y Tinling 5 observaron zonas de rotura de lámina basal en colesteatomas espontáneos einducidos en animales de experimentación y presencia de seudópodos epiteliales en lámina propia. Estos seudópodos epiteliales formaban conos epiteliales y eventualmente microcolesteatomas. Además se vio células inflamatorias como neutrófilos asociadas a estos sitios de rotura en la lámina basal. Kim et al ${ }^{10}$ observaron que la expresión de citoqueratina (proteína de filamento intermedio de origen epitelial) se altera en puntos focales, adyacentes al colesteatoma, y no en el colesteatoma mismo. Sugiriendo que el colesteatoma sería resultado de un proceso alterado de migración y actividad de los queratinocitos.

Sin que ninguna de las teorías clásicas sea suficiente, algunos investigadores han reunido conceptos de varias, es así como recientemente
Sudhoff y Tos $^{11}$ unificaron la teoría de la invaginación y la de las células basales. Su teoría proporciona evidencia para explicar la transición entre un bolsillo de retracción y un colesteatoma activo. Basados en hallazgos clínicos e inmunológicos plantean 4 etapas en la patogénesis del colesteatoma originado a partir de un bolsillo de retracción: (1) Elapa de bolsillo de retracción, (2) etapa de proliferación de bolsillo, (3) etapa de expansión de bolsillo y (4) etapa de reabsorción ósea.

Hasta aquí la teoría de Sudhoff y Tos explica la formación del bolsillo y su transformación en colesteatoma, sin embargo, las interrogantes sobre los gatillantes de los procesos de proliferación y migración celular aún quedan en pie.

\section{Barrera de conocimiento}

日 colesteatoma tiene un patrón de crecimiento alterado, diferente al del epitelio epidérmico normal, caracterizado por un crecimiento excesivo de queratinocitos que lleva a la destrucción de la mucosa. Este comportamiento frecuentemente se acompaña de inflamación, formación de tejido de granulación y destrucción ósea, hechos que determinan la agresividad del colesteatoma. Por mucho tiempo la destrucción ósea se atribuyó a la actividad inflamatoria de la perimatriz. Hoy en día se sabe que esta destrucción se debe a la expresión de ciertas citoquinas liberadas por los queratinocitos de la matriz y perimatriz como la interleuquina 1,6 y 8 , factor de necrosis tumoral y factor de crecimiento transformante ${ }^{12-15}$. También se ha visto que el factor de necrosis tumoral estimula la proliferación celular ${ }^{15}$, de esta forma la destrucción ósea podría estar ligada también a la proliferación celular por medio de las citoquinas liberadas por la matriz.

En 1992 Cattoretti el al ${ }^{16}$ describen por primera vez una técnica para cuantificar la proliferación celular que es altamente reproducible y fácil de realizar. Se trata del MIB1, un anticuerpo monoclonal dirigido contra el antígeno nuclear Ki-67. Este antígeno se expresa en el núcleo celular durante la fase S, G2, M y el término de la fase G1 del ciclo celular, y está ausente durante GO y el 
resto de G1. La cantidad de Ki-67 detectada en la capa superficial y basal de la matriz es, entonces, un índice confiable y ampliamente utilizado de la proliferación celular. Tiene la ventaja, frente a muchos otros métodos utilizados para evaluar la cinética celular (como determinación histológica de mitosis, citometría de flujo y autorradiografía de muestras expuestas a timidina tritiada entre otros), que detecta a todas las células en etapa de proliferación, y no sólo a las que se encuentran en fase S, de tal forma entrega información más veraz.

En 1996 Bujía et al ${ }^{17}$ evaluó las características de crecimiento de 20 colesteatomas en niños utilizando MIB1, comparándolas con el crecimiento de 15 colesteatomas adultos y 15 muestras de piel normal del meato auditivo de adultos. Las muestras de piel normal mostraron un puntaje promedio de MIB1 de 9,2\%, las de colesteatomas adultos de $28,2 \%$ y las de colesteatomas de niños un $42 \%$, siendo la diferencia estadísticamente significativa en todos los casos. Estos resultados sugieren una explicación para la conocida mayor agresividad clínica de los colesteatomas en niños. No se encontró diferencia entre los colesteatomas infectados y los no infectados, sugiriendo que este comportamiento es independiente de la contaminación bacteriana Sin embargo, el estudio no describe los métodos utilizados para certificar la infección ni con cuánta dedicación se buscó.

Mallet et $\mathrm{al}^{18}$ en 2003 publicaron un estudio en el que compararon la actividad proliferativa de 91 colesteatomas determinada por MIB1, con características clínicas y quirúrgicas de éstos. Establecieron dos grupos de colesteatomas según su actividad proliferativa: Los "activos", en los que todas las células basales estaban en división celular (todas las células basales presentaban inmunomarcación), y los "inactivos", en los que la marcación era discontinua. Compararon estos dos grupos con el grado de destrucción ósea, inflamación, edad del paciente, origen del colesteatoma (congénito o adquirido), punto de entrada del colesteatoma al oído medio y presencia de pólipo inflamatorio. Encontraron que los colesteatomas "activos" se asociaban en forma estadísticamente significativa a mayor destrucción ósea e inflamación de la caja timpánica. También encontraron mayor prevalencia de colesteatomas activos en niños, en quienes se sabe son más agresivos. Este trabajo muestra una clara asociación entre la alta actividad proliferativa del colesteatoma y la agresividad de éste, sugiriendo que además de la inflamación en la perimatriz, la matriz por sí sola puede provocar destrucción ósea Oro aspecto importante a destacar es la ventana que se abre al uso del MIB1 como una herramienta útil para predecir la agresividad del colesteatoma, su dificultad quirúrgica y tendencia a la recurrencia.

Una característica de los colesteatomas infectados es su persistencia y la tendencia de la infección a recurrir siendo la erradicación quirúrgica la única solución efectiva. Sin embargo, la falta de congruencia entre hallazgos quirúrgicos de colesteatomas muy agresivos y la falta de infección han hecho buscar la presencia de infecciones atípicas, es así como algunos autores plantean la teoría de los biofilms. Las bacterias pueden existir en la naturaleza de dos formas: como células móviles que se replican (forma planctónica), o como células quiescentes, adheridas a superficies, dentro de una matriz hidratada de polisacáridos y proteínas conocidas como biofims (forma Cecil). Este biofilm proporciona a las bacterias un ambiente que las protege de las condiciones externas adversas. Dentro de él, las bacterias tienen una alta actividad metabólica, sin embargo, no se replican lo que impide cultivarlas por métodos corrientes. Además, son altamente resistentes a los antibióticos, por mecanismos diferentes a los habituales y pueden pasar de este biofilm a la forma móvil tornándose infectantes. Bacterias como Pseudomonas y Staphylococcus aisladas frecuentemente de colesteatomas han demostrado tener capacidad de formar biofilms. 日 año 2002, Chole et al demostraron la presencia de biofilms mixtos (formados por gram positivos y negativos) en acúmulos de queratina en 21 de 22 colesteatomas de ratones de experimentación y en 16 de 24 colesteatomas humanos. Estos podrían explicar las características de los colesteatomas infectados: la persistencia y la recurrencia de infección, a pesar de no presentarse como una infección clásica.

Ronchetti et al ${ }^{19}$ opinan que las bacterias encontradas habitualmente en los colesteatomas no 
explican el típico estado inflamatorio de éstos. Considerando que Chlamidia pneumoniae es un patógeno intracelular nunca antes estudiado en colesteatomas y que se le ha vinculado con varias enfermedades que cursan con inflamación crónica como el asma, arteriosclerosis e incluso la enfermedad de Alzheimer, Ronchetti propuso estudiarlo. Estudió 32 colesteatomas humanos obteniendo muestras de sus tres regiones: contenido, matriz y perimatriz. Realizó reacción de polimerasa en cadena para $C$ pneumoniae, $H$ influenzae y $M$ pneumoniae, con dos técnicas diferentes para cada muestra. Detectó presencia de $C$ pneumoniae en $50 \%$ de los colesteatomas, en 2 casos encontró presencia de $H$ influenzae y en ningún caso $M$ pneumoniea. Esta constituye la primera demostración de $C$ Pneumoniae en colesteatomas humanos y sugiere una relación de causalidad directa entre la acción del patógeno y la formación del colesteatoma. En todos los casos, con la excepción de tres, el patógeno se encontró sólo en la matriz del colesteatoma lo que indica que su localización en regiones está bien definida. Es más, esto sugiere que el patógeno se asocia con las primeras etapas en la formación del colesteatoma.

Fnalmente Choufani et al ${ }^{20}$ estudiaron en 56 colesteatomas la expresión del factor inhibidor de migración de macrófagos (en inglés MIF). Observó mayor presencia en epitelio y vasos sanguíneos de tejido conectivo en oídos con colesteatoma recurrente. Su expresión fue independiente del estado infeccioso del colesteatoma. También se vio mayor expresión de MIF en muestras que sobreexpresaban genes encargados de la diferenciación celular, regulación del crecimiento e invasión (galectina, catepsinas y metaloproteinasas). Concluyó finalmente que la expresión de MIF se correlaciona con la agresividad y menor diferenciación del colesteatoma, tanto medida clínicamente (recurrencia), como genéticamente (galectina metaloproteinasas, etc).

\section{BIBLIOGRAFÍA}

1. SADE J, Halevy A. The etiology of bone destruc- tion in chronic otitis media. $J$ Laryngol Otol 1974, 88: 139-43.

2. CHOLE RA. Cellular and subcellular events of bone resorption in human and experimental cholesteatoma: the role of osteoclasts. Laryngoscope 1984; 94: 76-95.

3. LeVenson MJ, Micha日es L, PARISIERS. Congenital cholesteatomas of the middle ear in children: origin and management. Otolaryn Cinics NAm 1989; 22(5): 941-53.

4. SADEJ, HaleVY A. The natural history of chronic otitis media. J Laryngol Otol 1976; 90: 743-51.

5. CHOLE RA, TINLING SP. Basal lamina breaks in the histogenesis of cholesteatoma. Laryngoscope 1985; 95: 270-5.

6. Poeds, Abou-Halawa A, Abde-Razek O. Analysis of the dysfunctional eustachian tube by video endoscopy. Otol Neurotol 2001; 22: 5905.

7. SADEJ, FUCHS C, LUNTZM. Shrapnell membrane and mastoid pneumatization. Arch Otolaryngol Head Neck Surg 1997; 123: 584-8.

8. SADE J. Hyperectasis: the hyperinflated tympanic membrane: the middle ear as an actively controlled system. Otol Neurotol 2001; 22: 133-9.

9. HaSme S, TaKaHaSH H, Honno I et al. Mastoid condition and clinical course of cholesteatoma. ORL J Otorhinolaryngol Relat Spec 2001; 63: 160-4.

10. KIM HJ, TINLING SP, OHOLE RA. Expression patterns of cytokeratins in retraction pocket cholesteatomas. Laryngoscope 2001; 111: 1032-6.

11. SUDHOF H, Tos M. Pathogenesis of attic cholesteatoma: clinical and immunohistochemical support for combination of retraction theory and proliferation theory. Am J Ool 2000; 21: 786-92.

12. AMAR MS, WISHAHI HF, ZAKHARY MM. Ginical and biochem-ical studies of bone destruction in cholesteatoma J Laryn-gol Otol 1986; 110: 534-9.

13. MARENDA SA, AUPDEMORTE TB. Localization of cytokines in cholesteatoma tissue. Otolaryngol Head Neck Surg 1995; 112: 359-68. 
14. PARK Y, JUN BH, OHO IH. Expression of the cytokine network and adhesion molecules in aural cholesteatoma. Sixth In-ternational Conference on Cholesteatoma Ear Surgery, Cannes, France, June 2000.

15. YAN S, HUANG C. Tumor necrosis factor alpha in middle ear cholesteatoma and its effect on keratinocytes in vitro. Ann Otol Rhinol Laryngol 1991; 100: 157-61.

16. CATTORETII G, BECKER MHG, KEY G Monoclonal antibodies against recombinant parts of the $\mathrm{K}$ 67 antigen (Mib 1 and Mib3) detect proliferating cells in microwave-processed formalin-fixed paraffin sections. J Pathol 1992; 168: 357-63.

17. BuJia J, HOLly A, ANTOLI-Candea F, GuzMán TAPIA M, KastenBaUer E Immunobiological peculiarities of cholesteatoma in children: quantification of epithelial proliferation by MIB1. Laryngoscope 1996; 106: 865-8.
18. Mallet Y, Nouwen J, LeoOMte-Houcke M, DesAULTY A. Aggresiveness and quantification of epithelial proliferation of middle ear cholesteatoma by MIB1. Laryngoscope 2003; 113: 32831.

19. RONCETII F, RONCETII R, GugLIeIMI F. Detection of Chlamydia pneumoniae in cholesteatoma tissue; Any pathologenetic rol? Otol and Neuotol 2003; 24: 353-7.

20. GHOUfANI G, GHaNOONI R, DECAESTECKER Cet aL. Detection of macrophage migration inhibitory factor (MIF) in human cholesteatomas and functional implications of correlations to recurrence status and to expression of matrix metaIloproteinases-3/9, retinoic acid receptor-b, and anti-apoptotic galectin-3. Laryngoscope 2001; 111: 1656-62.

21. Tos M, POULSEN G Attic retractions following secretory otitis. Acta Aolaryngol 1980; 89: 479-86. 